\title{
Vector-valued modular functions for the modular group and the hypergeometric equation
}

\author{
Peter Bantay and Terry Gannon
}

\begin{abstract}
A general theory of vector-valued modular functions, holomorphic in the upper half-plane, is presented for finite-dimensional representations of the modular group. This also provides a description of vector-valued modular forms of arbitrary half-integer weight. It is shown that the space of these modular functions is spanned, as a module over the polynomials in $J$, by the columns of a matrix that satisfies an abstract hypergeometric equation, providing a simple solution of the Riemann-Hilbert problem for representations of the modular group. Restrictions on the coefficients of this differential equation implied by analyticity are discussed, and an inversion formula is presented that allows the determination of an arbitrary vector-valued modular function from its singular behavior. Questions of rationality and positivity of expansion coefficients are addressed. Closed expressions for the number of vector-valued modular forms of half-integer weight are given, and the general theory is illustrated on simple examples.
\end{abstract}

\section{Introduction}

The notions of modular functions and forms — and their generalizations are among the most fruitful in all of mathematics, and with the arrival of string theory they have become standard fare in mathematical physics as well. Vector-valued modular functions $\mathbb{X}(\tau)$ for $\mathrm{SL}_{2}(\mathbb{Z})$ appear, for instance, as characters of vertex operator algebras [17] and conformal field theories [6], and in the Norton series of Generalized Moonshine [14]; moreover, in rational conformal field theory (RCFT), vector-valued modular forms of arbitrary rational weight appear as conformal blocks on a once-punctured torus. In spite of its importance, there has been little attempt at a systematic treatment of this theory $([11,7]$ are exceptions).

In these contexts, singularities of the component functions $\mathbb{X}_{\eta}(\tau)$ appear at the cusps $\mathbb{Q} \cup\{\infty\}$, but not in the upper half-plane $\mathbf{H}$, and we will restrict our attention to such functions. In a previous paper [4], we explained (with examples) how to obtain all such vector-valued modular functions, 
given the corresponding multiplier $\rho$, a finite-dimensional representation of $(\mathrm{P}) \mathrm{SL}_{2}(\mathbb{Z})$. In this paper, we focus on the underlying structure of these spaces of vector-valued modular functions. They are generated by the $\mathrm{SL}_{2}(\mathbb{Z})$-Hauptmodul $J(\tau)$, together with the columns of a certain fundamental matrix $\boldsymbol{\Xi}(\tau)$. We explain how everything is conveniently recovered from the exponents $\boldsymbol{\Lambda}$ at infinity and a numerical matrix $\mathcal{X}$ (essentially, the first non-trivial $q$-coefficients of $\boldsymbol{\Xi}(\tau))$. The other $q$-coefficients of $\boldsymbol{\Xi}(\tau)$ can be obtained from a differential equation, the monodromy of which is determined by $\rho$. Our results extend directly to vector-valued modular forms of half-integer weight: for instance, we obtain an explicit formula for the dimension of the spaces of such forms.

In Section 2, we review the framework of [4], and discuss a subtlety: the choice of integer part of the exponent matrix $\boldsymbol{\Lambda}$. Section 3 explains how the differential equation satisfied by the fundamental matrix may be recast into an abstract hypergeometric equation, and the consequences this has on the various quantities involved. Section 4 gives some concrete examples, illustrating the effectiveness of our results. Section 5 provides an inversion formula, which allows the explicit computation of any vector-valued modular function from its singular part, provided the fundamental matrix is known. In the motivating examples, the $q$-expansions have non-negative integer coefficients. Section 6 explains how the existence of such $q$-expansions constrains $\rho$. The appendix describes what happens when - as is typical in vertex operator algebras or conformal field theory - $\rho$ is a representation of $\mathrm{SL}_{2}(\mathbb{Z})$ rather than of $\mathrm{PSL}_{2}(\mathbb{Z})$.

\section{The fundamental matrix}

Consider a matrix representation $\rho: \mathrm{SL}_{2}(\mathbb{Z}) \rightarrow \mathrm{GL}_{d}(\mathbb{C})$ whose kernel contains $\left(\begin{array}{rr}-1 & 0 \\ 0 & -1\end{array}\right)$, and for which $T=\rho\left(\begin{array}{ll}1 & 1 \\ 0 & 1\end{array}\right)$ is a diagonal matrix of finite order. We associate to $\rho$ the set $\mathcal{M}(\rho)$ of all those maps $\mathbb{X}: \mathbf{H} \rightarrow \mathbb{C}^{d}$ which are holomorphic in the upper half-plane $\mathbf{H}=\{\tau \mid \operatorname{Im} \tau>0\}$, transform according to $\rho$, that is ${ }^{1}$

$$
\mathbb{X}\left(\frac{a \tau+b}{c \tau+d}\right)=\rho\left(\begin{array}{ll}
a & b \\
c & d
\end{array}\right) \mathbb{X}(\tau)
$$

\footnotetext{
${ }^{1}$ Here and in what follows we view $\mathbb{X}(\tau)$ as a column vector.
} 
for all $\left(\begin{array}{ll}a & b \\ c & d\end{array}\right) \in \mathrm{SL}_{2}(\mathbb{Z})$ and $\tau \in \mathbf{H}$, and have only finite order poles at the cusps [4]. This last condition means the following: since $\rho\left(\begin{array}{ll}1 & 1 \\ 0 & 1\end{array}\right)$ is diagonal of finite order, there exists a diagonal matrix $\boldsymbol{\Lambda}$ (the exponent matrix) such that

$$
\rho\left(\begin{array}{ll}
1 & 1 \\
0 & 1
\end{array}\right)=\exp (2 \pi i \boldsymbol{\Lambda})
$$

the diagonal elements of $\boldsymbol{\Lambda}$ being rational numbers. Because of Equation (2.1), the map $\exp (-2 \pi i \tau \boldsymbol{\Lambda}) \mathbb{X}(\tau)$ is periodic in $\tau$ (with period 1 ): consequently, it may be expanded into a Fourier series ${ }^{2}$

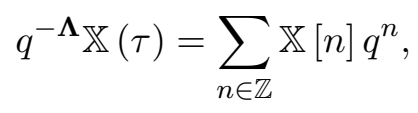

where $q=\exp (2 \pi i \tau)$. We define the principal part $\mathcal{P} \mathbb{X}$ of $\mathbb{X}$ as the sum of the terms with negative powers of $q$ on the RHS of Equation (2.3), i.e.,

$$
\mathcal{P X}(q)=\sum_{n<0} \mathbb{X}[n] q^{n}
$$

With this definition, $\mathbb{X}$ has finite order poles at the cusps if and only if its principal part $\mathcal{P X}$ is a finite sum.

Clearly, the space $\mathcal{M}(\rho)$ is an infinite-dimensional linear space over $\mathbb{C}$, a basis being provided by the maps $\mathbb{X}^{(\xi ; n)} \in \mathcal{M}(\rho)$ which have a pole of order $n>0$ at the $\xi$ th position, i.e.,

$$
[\mathcal{P X}(\xi ; n)(q)]_{\eta}=q^{-n} \delta_{\xi \eta}
$$

We call these $\mathbb{X}^{(\xi ; n)}$ the canonical basis vectors; they are clearly linearly independent, and that they exist and therefore span $\mathcal{M}(\rho)$ was explained in [4] (an independent proof is provided at the end of Section 3).

\footnotetext{
${ }^{2}$ In all what follows, we shall alternate freely between the notations $f(\tau)$ and $f(q)$ for one and the same quantity $f$ : in general, the notation $f(\tau)$ is meant to emphasize that we consider $f$ as a (holomorphic) function on the upper half-plane $\mathbf{H}$, while $f(q)$ refers to its expansion as a power series in $q=\exp (2 \pi i \tau)$.
} 
Let

$$
J(\tau)=q^{-1}+\sum_{n=1}^{\infty} c(n) q^{n}=q^{-1}+196884 q+\cdots
$$

denote the Hauptmodul of $\mathrm{SL}_{2}(\mathbb{Z})$, i.e., the (suitably normalized) generator of the field of modular functions for $\mathrm{SL}_{2}(\mathbb{Z})$ (for this and other aspects of the classical theory of modular functions and forms, see e.g., [1]). Multiplication by $J$ takes the space $\mathcal{M}(\rho)$ to itself, in other words $\mathcal{M}(\rho)$ is a $\mathbb{C}[J]$-module. The important point is that this is a (free) $\mathbb{C}[J]$-module of finite rank, because the canonical basis vectors satisfy the recursion relations [4]

$$
\mathbb{X}^{(\xi ; m+1)}=J(\tau) \mathbb{X}^{(\xi ; m)}-\sum_{n=1}^{m-1} c(n) \mathbb{X}^{(\xi ; m-n)}-\sum_{\eta} \mathcal{X}_{\eta}^{(\xi ; m)} \mathbb{X}^{(\eta ; 1)}
$$

where

$$
\mathcal{X}_{\eta}^{(\xi ; m)}=\mathbb{X}^{(\xi ; m)}[0]_{\eta}=\lim _{q \rightarrow 0}\left(\left[q^{-\Lambda_{\mathbb{X}}}(\xi ; m)(q)\right]_{\eta}-q^{-m} \delta_{\xi \eta}\right)
$$

denotes the "constant part" of $\mathbb{X}^{(\xi ; m)}$. These recursion relations allow to express each canonical basis vector $\mathbb{X}^{(\xi ; m)}$ in terms of the $\mathbb{X}^{(\xi ; 1)}$ s, proving that the latter generate the $\mathbb{C}[J]$-module $\mathcal{M}(\rho)$. Later on, we will give an explicit expression - Equation $(5.2)$ - for the $\mathbb{X}^{(\xi ; m)}$ s. We will see shortly that the $\mathbb{X}^{(\xi ; 1)}$ are linearly independent over the field $\mathbb{C}(J)$ of modular functions, and thus the $\mathbb{C}[J]$-module $\mathcal{M}(\rho)$ has rank $d$.

Besides the recursion relations Equation (2.7), there is a second set of relations — the differential relations [4] — between the canonical basis vectors. They follow from the fact that the differential operator

$$
\nabla=\frac{\mathcal{E}(\tau)}{2 \pi i} \frac{d}{d \tau}
$$

maps $\mathcal{M}(\rho)$ to itself, where

$$
\mathcal{E}(\tau)=\frac{E_{10}(\tau)}{\Delta(\tau)}=\sum_{n=-1}^{\infty} \mathcal{E}_{n} q^{n}=q^{-1}-240-141444 q-\cdots
$$

is the quotient of the (normalized) Eisenstein series of weight 10 by the discriminant form $\Delta(\tau)=q \prod_{n=1}^{\infty}\left(1-q^{n}\right)^{24}$ of weight 12 . Looking at the 
action of $\nabla$ on the canonical basis vectors, one gets the differential relations

$$
\nabla \mathbb{X}^{(\xi ; m)}=\left(\boldsymbol{\Lambda}_{\xi \xi}-m\right) \sum_{n=-1}^{m-1} \mathcal{E}_{n} \mathbb{X}^{(\xi ; m-n)}+\sum_{\eta} \boldsymbol{\Lambda}_{\eta \eta} \mathcal{X}_{\eta}^{(\xi ; m)} \mathbb{X}^{(\eta ; 1)}
$$

The compatibility of the recursion and differential relations requires that ${ }^{3}$

$$
\nabla \mathbb{X}^{(\xi ; 1)}=(J-240)\left(\boldsymbol{\Lambda}_{\xi \xi}-1\right) \mathbb{X}^{(\xi ; 1)}+\sum_{\eta}\left(1+\boldsymbol{\Lambda}_{\eta \eta}-\boldsymbol{\Lambda}_{\xi \xi}\right) \mathcal{X}_{\eta}^{(\xi ; 1)} \mathbb{X}^{(\eta ; 1)}
$$

which is a first-order ordinary differential equation — the compatibility equation - for the $\mathbb{X}^{(\xi ; 1)} \mathrm{s}$.

One may recast the compatibility equation, Equation (2.12), in a more suggestive form by introducing the fundamental matrix

$$
\Xi(\tau)_{\xi \eta}=\left[\mathbb{X}^{(\eta ; 1)}(\tau)\right]_{\xi}
$$

whose columns span over $\mathbb{C}[J]$ the module $\mathcal{M}(\rho)$. Then Equation (2.12) takes the form

$$
\frac{1}{2 \pi i} \frac{d \boldsymbol{\Xi}(\tau)}{d \tau}=\boldsymbol{\Xi}(\tau) \mathfrak{D}(\tau)
$$

where

$$
\mathfrak{D}(\tau)=\frac{1}{\mathcal{E}(\tau)}\{(J(\tau)-240)(\boldsymbol{\Lambda}-1)+\mathcal{X}+[\boldsymbol{\Lambda}, \mathcal{X}]\}
$$

and $\mathcal{X}_{\xi \eta}=\mathcal{X}_{\xi}^{(\eta ; 1)}$ is the so-called characteristic matrix (as usual, $[\mathcal{X}, \boldsymbol{\Lambda}]=$ $\mathcal{X} \boldsymbol{\Lambda}-\boldsymbol{\Lambda} \mathcal{X}$ denotes the commutator of matrices). Note that Equation (2.14) has singular points at the poles of $\mathfrak{D}(\tau)$, i.e., at the $\mathrm{SL}_{2}(\mathbb{Z})$-orbits of the cusp $\tau=i \infty$ and elliptic points $\tau=i$ and $\tau=\exp (2 \pi i / 3)$. Taking into account the boundary condition

$$
q^{1-\boldsymbol{\Lambda}_{\xi \xi}} \boldsymbol{\Xi}(q)_{\xi \eta}=\delta_{\xi \eta}+\mathrm{O}(q) \quad \text { as } \quad q \rightarrow 0,
$$

which follows from Equation (2.5), one can solve Equation (2.14), provided one knows the exponent matrix $\boldsymbol{\Lambda}$ and the characteristic matrix $\mathcal{X}$, determining then from Equation $(2.7)$ the canonical basis vectors $\mathbb{X}^{(\xi ; m)}$. The

\footnotetext{
${ }^{3}$ We show, at the end of Section 5 , that this is not only a necessary, but also a sufficient condition for the compatibility of the recursion and differential relations.
} 
theory of ordinary differential equations guarantees Equation (2.14) to have series solutions that converge in suitably small neighborhoods of $\mathbf{H}$ avoiding the elliptic points, but the holomorphicity of $\boldsymbol{\Xi}(\tau)$ implies that those series actually converge throughout $\mathbf{H}$.

Equation (2.16) tells us that the determinant $\operatorname{det} \boldsymbol{\Xi}(\tau)$ has leading term $q^{\operatorname{Tr}(\boldsymbol{\Lambda}-1)}$ as $q \rightarrow 0$, and so is not identically 0 . Thus, its columns $\mathbb{X}^{(\xi ; 1)}$ are indeed linearly independent over $\mathbb{C}(J)$. This invertibility of $\boldsymbol{\Xi}(\tau)$ legitimates its appellation, since it is now seen as a fundamental solution of Equation (2.14).

Actually, the results so far enable us already to discuss vector-valued modular forms of half-integer weight for $\mathrm{SL}_{2}(\mathbb{Z})$. By a modular form of weight $k \in 1 / 2 \mathbb{Z}$ for the (possibly projective) $\operatorname{PSL}_{2}(\mathbb{Z})$-representation $\varrho$ we will mean a map $\mathbb{X}: \mathbf{H} \rightarrow \mathbb{C}^{d}$ that is holomorphic everywhere in $\mathbf{H}$, transforms according to

$$
\mathbb{X}\left(\frac{a \tau+b}{c \tau+d}\right)=(c \tau+d)^{k} \varrho\left(\begin{array}{ll}
a & b \\
c & d
\end{array}\right) \mathbb{X}(\tau),
$$

and which tends to a finite limit as $\tau \rightarrow i \infty$. Such an $\mathbb{X}$ is a cusp form if it vanishes at $\tau=i \infty$. As before, we require $\varrho\left(\begin{array}{ll}1 & 1 \\ 0 & 1\end{array}\right)$ to be diagonal and of finite order. We will denote by $M_{k}(\varrho)$ and $S_{k}(\varrho)$ the space of vector-valued modular forms (resp. cusp forms) of weight $k$ for the representation $\varrho$ : clearly, the latter is a subspace of the former. When $k \notin \mathbb{Z}, \varrho$ will be a true (as opposed to merely projective) representation of the metaplectic group $\mathrm{Mp}_{2}(\mathbb{Z})$, a double-cover of $\mathrm{SL}_{2}(\mathbb{Z})$; when $k$ is odd, $\varrho$ will be a true representation of $\mathrm{SL}_{2}(\mathbb{Z})$, sending $\left(\begin{array}{cc}-1 & 0 \\ 0 & -1\end{array}\right)$ to minus the identity; when $k$ is even, $\varrho$ is a true representation of $\mathrm{PSL}_{2}(\mathbb{Z})$. Note that when $\varrho$ is the trivial representation, we recover the classical theory of modular forms of even weight.

Let $\eta(\tau)=q^{1 / 24} \prod_{n=1}^{\infty}\left(1-q^{n}\right)$ be the Dedekind eta function, and let $\mu$ denote its multiplier (see, e.g., [10, Chapter 4] for a formula for $\mu$ ). Then, for any $k \in 1 / 2 \mathbb{Z}$ we have a natural injective map

$$
\begin{aligned}
\phi: M_{k}(\varrho) & \rightarrow \mathcal{M}\left(\varrho \otimes \mu^{-2 k}\right), \\
\mathbb{X} & \mapsto \eta^{-2 k} \mathbb{X},
\end{aligned}
$$

which allows to embed the space $M_{k}(\varrho)$ of modular forms of weight $k$ into the module $\mathcal{M}\left(\varrho \otimes \mu^{-2 k}\right)$. It is easy to verify that the space $M_{k}(\varrho)$ will be trivial unless $\rho=\varrho \otimes \mu^{-2 k}$ is a true representation of $\mathrm{PSL}_{2}(\mathbb{Z})$, in which case $\mathcal{M}(\rho)$ is covered by the analysis of this paper. Non-singularity of $\mathbb{X} \in M_{k}(\varrho)$ as $\tau \rightarrow i \infty$ bounds the order of the pole of the singular part of 
$\eta^{-2 k} \mathbb{X}$; as a result, the spaces $M_{k}(\varrho)$ (hence $S_{k}(\varrho)$ too) are finite-dimensional, and explicit bases can be found. As for their dimension, one obtains the result

$$
\begin{aligned}
\operatorname{dim} M_{k}(\varrho) & =\max \left(0, \operatorname{Tr}\left[\boldsymbol{\Lambda}+\frac{k}{12}\right]\right) \\
\operatorname{dim} S_{k}(\varrho) & =\max \left(0,-\operatorname{Tr}\left[1-\frac{k}{12}-\boldsymbol{\Lambda}\right]\right)
\end{aligned}
$$

where $\boldsymbol{\Lambda}$ denotes the exponent matrix of $\rho=\varrho \otimes \mu^{-2 k}$ and $[x]$ denotes the integer part of $x$ ( $x$ can be a matrix): note that $\boldsymbol{\Lambda}$ varies with the weight $k$.

When $\varrho$ is the trivial representation, Equation (2.19) reduces to classical results for the dimensions of modular and cusp forms for $\mathrm{SL}_{2}(\mathbb{Z})$. Those equations also lead to the following expressions for the trace of the integer part of $\boldsymbol{\Lambda}$ (for a true $\mathrm{PSL}_{2}(\mathbb{Z})$ representation $\varrho$ ):

$$
\operatorname{Tr}[1-\boldsymbol{\Lambda}]=\operatorname{dim} M_{2}(\bar{\varrho})
$$

and

$$
\operatorname{Tr}[\boldsymbol{\Lambda}]=\operatorname{dim} M_{0}(\varrho)-\operatorname{dim} S_{2}(\bar{\varrho})
$$

where $\bar{\varrho}$ denotes the contragredient representation of $\varrho$. We leave the derivation of these results - which amount to careful book-keeping - to a future publication. Equation (2.19) recovers and generalizes the dimension formula in [7], which was proved using the Eichler-Selberg trace formula.

At this point we should make an important proviso: Equation (2.2) only determines the fractional part of the diagonal elements of the exponent matrix, not their integer part. This is important, since the values of these integer parts enter the definition Equation (2.4) of the principal part map $\mathcal{P}$, and hence of the canonical basis vectors $\mathbb{X}^{(\xi ; n)}$. Another choice of these integer parts leads to a different set of canonical basis vectors, hence different characteristic and fundamental matrices, while $\mathcal{M}(\rho)$ remains unchanged. Even more important is the observation that for an arbitrary choice of the integer part of $\boldsymbol{\Lambda}$, the principal part map $\mathcal{P}$ may not be injective (i.e., the terms singular with respect to $\boldsymbol{\Lambda}$ may not determine the functions) and may not be surjective (i.e., not all canonical basis vectors may exist). As we are 
going to explain, one can choose the integer part of the exponent matrix at will, provided that the relation ${ }^{4}$

$$
\operatorname{Tr}(\boldsymbol{\Lambda})=\frac{5 d}{12}+\frac{1}{4} \operatorname{Tr}(S)+\frac{2}{3 \sqrt{3}} \operatorname{Re}\left(e^{-\pi i / 6} \operatorname{Tr}(U)\right)
$$

holds, where $d$ is the dimension of $\rho$, and we use the notations

$$
S=\rho\left(\begin{array}{cc}
0 & -1 \\
1 & 0
\end{array}\right) \quad \text { and } \quad U=\rho\left(\begin{array}{cc}
0 & -1 \\
1 & -1
\end{array}\right) .
$$

Surjectivity fails if $\operatorname{Tr}(\boldsymbol{\Lambda})$ is greater than the RHS of Equation (2.22); injectivity fails if it is less.

To see how this comes about, first note that $\mathcal{P}$ is invertible iff both $\boldsymbol{\Xi}$ exists and its columns span $\mathcal{M}(\rho)$ over $\mathbb{C}[J]$, iff those of $\boldsymbol{\Xi}$ do. Suppose that $\mathcal{X}_{12} \neq 0$, and consider the matrix

$$
M(\tau)=\left(\begin{array}{ccccc}
0 & -\mathcal{X}_{12} & 0 & \cdots & 0 \\
\frac{1}{\mathcal{X}_{12}} & J(\tau)-C & -\frac{\mathcal{X}_{13}}{\mathcal{X}_{12}} & \cdots & -\frac{\mathcal{X}_{1 d}}{\mathcal{X}_{12}} \\
0 & -\mathcal{X}_{32} & 1 & 0 & 0 \\
\vdots & \vdots & 0 & \ddots & 0 \\
0 & -\mathcal{X}_{d 2} & 0 & 0 & 1
\end{array}\right),
$$

where $C$ is a suitable constant. All matrix elements of $M$ belong to $\mathbb{C}[J]$, and the same holds for the inverse matrix $M^{-1}$, since det $M=1$ irrespectively of the value of the constant $C$. Consequently, the columns of the matrix $\boldsymbol{\Xi}^{\prime}(\tau)=\boldsymbol{\Xi}(\tau) M(\tau)$ span $\mathcal{M}(\rho)$ over $\mathbb{C}[J]$, iff those of $\boldsymbol{\Xi}$ do. By a suitable choice of the constant $C$ one can achieve that $\boldsymbol{\Xi}^{\prime}(\tau)$ satisfies the boundary condition Equation (2.16) with

$$
\boldsymbol{\Lambda}^{\prime}=\boldsymbol{\Lambda}+\left(\begin{array}{ccccc}
1 & & & & \\
& -1 & & & \\
& & 0 & & \\
& & & \ddots & \\
& & & & 0
\end{array}\right)
$$

\footnotetext{
${ }^{4}$ Here we assume that the matrix representation $\rho$ is indecomposable, i.e., cannot be written as the direct sum of two matrix representations (this holds for any representation coming from, e.g., RCFT, as every entry of the vacuum column of $S$ will necessarily be non-zero): otherwise, one should apply these considerations to each direct summand separately. Note that these statements are basis-dependent: we are certainly not claiming that the representation $\rho$ is irreducible.
} 
This means that $\boldsymbol{\Xi}^{\prime}(\tau)$ is also a fundamental matrix for $\mathcal{M}(\rho)$, corresponding to the exponent matrix $\boldsymbol{\Lambda}^{\prime}$.

By applying a suitable sequence of transformations of the above type, we see that one can add to $\boldsymbol{\Lambda}$ any integral diagonal matrix whose trace vanishes. But can we alter the trace of $\boldsymbol{\Lambda}$ as well? The answer is no, for we will see in Section 3 (when we will have all the necessary tools at our disposal) that the invertibility of $\mathcal{P}$ implies Equation (2.22).

In summary, the structure of the $\mathbb{C}[J]$-module $\mathcal{M}(\rho)$ is completely determined by the fundamental matrix $\boldsymbol{\Xi}(\tau)$, once an exponent matrix $\boldsymbol{\Lambda}$ satisfying Equations (2.2) and (2.22) has been chosen. The fundamental matrix is itself completely determined by the pair $(\boldsymbol{\Lambda}, \mathcal{X})$ of exponent and characteristic matrices, namely as the solution of the compatibility equation Equation (2.14) satisfying the boundary condition Equation (2.16). For this reason, we consider the pair $(\boldsymbol{\Lambda}, \mathcal{X})$ as the basic data characterizing the representation $\rho$.

For example, the representation $\rho$ may be recovered from the compatibility equation. Indeed, Equation (2.14) is invariant under modular transformations

$$
\tau \mapsto \frac{a \tau+b}{c \tau+d}
$$

for $\left(\begin{array}{ll}a & b \\ c & d\end{array}\right) \in \mathrm{SL}_{2}(\mathbb{Z})$, which means that such a transformation takes a solution to another solution. Since the equation is linear, this new solution is of the form $M \boldsymbol{\Xi}(\tau)$ for some matrix $M \in \mathrm{GL}_{d}(\mathbb{C})$. Comparing this with Equations (2.1) and (2.13), and using the aforementioned invertibility of $\boldsymbol{\Xi}(\tau)$, we see that $M=\rho\left(\begin{array}{ll}a & b \\ c & d\end{array}\right)$, the matrix representing the given modular transformation, i.e.,

$$
\boldsymbol{\Xi}\left(\frac{a \tau+b}{c \tau+d}\right)=\rho\left(\begin{array}{ll}
a & b \\
c & d
\end{array}\right) \boldsymbol{\Xi}(\tau)
$$

But this argument works for any pair $(\boldsymbol{\Lambda}, \mathcal{X})$, i.e., any such pair determines a finite-dimensional representation $\rho$ via Equation (2.25). This seems to suggest that the pair $(\boldsymbol{\Lambda}, \mathcal{X})$ could be chosen at will, but this is not the case: the analyticity of the fundamental matrix $\boldsymbol{\Xi}(\tau)$ - namely, that it is single valued and holomorphic throughout the whole upper half-plane $\mathbf{H}$, including the elliptic points $\tau=i$ and $\tau=\exp (2 \pi i / 3)$ - puts severe restrictions on the pair $(\boldsymbol{\Lambda}, \mathcal{X})$. To understand these, it turns out to be convenient to transform the compatibility equation to an equivalent form, which is the subject of the next section. 


\section{The hypergeometric form of the compatibility equation}

Consider the function

$$
\mathfrak{z}(\tau)=\frac{984-J(\tau)}{1728},
$$

which maps the upper half-plane $\mathbf{H}$ onto the complex plane $\mathbb{C}$. Note that $\mathfrak{z}(i)=0$ and $\mathfrak{z}\left(e^{2 \pi i / 3}\right)=1$. As usual, we extend the definition of $\mathfrak{z}$ so that it maps $\tau=i \infty$ to $\infty$ (since $\mathfrak{z}$ has a first order pole at the cusp $\tau=i \infty) \cdot \mathfrak{z}$ is clearly modular invariant, i.e., it maps points on the same $\mathrm{SL}_{2}(\mathbb{Z})$ orbit to the same point of $\mathbb{C}$, and can thus be viewed as a map from $\mathbf{H} / \mathrm{SL}_{2}(\mathbb{Z})$ to $\mathbb{C}$. Viewed this way, it is one-to-one, and at the elliptic points $\tau=i$ and $\tau=\exp (2 \pi i / 3)$ it has valence 2 (respectively 3$)$ - this smooths the conical singularities of the modular curve $\mathbf{H} / \mathrm{SL}_{2}(\mathbb{Z})$. Finally, $\mathfrak{z}(\tau)$ satisfies the differential equation

$$
\nabla \mathfrak{z}=1728 \mathfrak{z}(\mathfrak{z}-1) .
$$

The simplest way to see that Equation (3.2) holds is to note that $\nabla \mathfrak{z}$ is modular invariant, holomorphic in $\mathbf{H}$, and has a pole of order 2 at $\tau=i \infty$, and hence it is a quadratic polynomial in $\mathfrak{z}$; moreover, it vanishes at the elliptic points because $E_{10}$ vanishes there. Equation (3.2) then follows by comparing the coefficients of $q^{-2}$.

Let us consider the fundamental matrix as a (multivalued) function of $\mathfrak{z}$. Then, by applying the chain rule and Equation (3.2), one arrives at the following form of the compatibility equation:

$$
\frac{d \boldsymbol{\Xi}(\mathfrak{z})}{d \mathfrak{z}}=\boldsymbol{\Xi}(\mathfrak{z})\left(\frac{\mathcal{A}}{2 \mathfrak{z}}+\frac{\mathcal{B}}{3(\mathfrak{z}-1)}\right)
$$

with

$$
\begin{aligned}
\mathcal{A} & =\frac{31}{36}(1-\boldsymbol{\Lambda})-\frac{1}{864}(\mathcal{X}+[\boldsymbol{\Lambda}, \mathcal{X}]), \\
\mathcal{B} & =\frac{41}{24}(1-\boldsymbol{\Lambda})+\frac{1}{576}(\mathcal{X}+[\boldsymbol{\Lambda}, \mathcal{X}]) .
\end{aligned}
$$

The important observation is that Equation (3.3) is an abstract hypergeometric equation, since it has three regular singular points (at $\mathfrak{z}=0,1$ and $\infty$ ), and much is known about the analytic properties of the solutions 
of Equation (3.3) (background for the following material is provided in, e.g., [8, Chapter 6]). As a function of $\mathfrak{z}$ the fundamental matrix is not single valued - its multivaluedness, i.e., the monodromy of Equation (3.3), is described by the representation $\rho$. In particular, the monodromies around $\mathfrak{z}=0, \mathfrak{z}=1, \mathfrak{z}=\infty$ are given by

$$
S=\rho\left(\begin{array}{cc}
0 & -1 \\
1 & 0
\end{array}\right), \quad U=\rho\left(\begin{array}{cc}
0 & -1 \\
1 & -1
\end{array}\right), \quad T=\rho\left(\begin{array}{ll}
1 & 1 \\
0 & 1
\end{array}\right)
$$

respectively. Because the residues of Equation (3.3) at these points are $\mathcal{A} / 2, \mathcal{B} / 3$ and $\boldsymbol{\Lambda}-1$, the matrices $S$ and $U$ are conjugate to $\exp (\pi i \mathcal{A})$ and $\exp (2 \pi i \mathcal{B} / 3)$, respectively, and one has $S U=T^{-1}=\exp (-2 \pi i \boldsymbol{\Lambda})$. We find that the monodromy group of the abstract hypergeometric equation Equation (3.3) is precisely the image of $\rho$.

Let us concentrate on the singular points $\mathfrak{z}=0$ and $\mathfrak{z}=1$ of Equation (3.3). The denominators 2 and 3 of the residues $\mathcal{A} / 2$ and $\mathcal{B} / 3$ match the valence of the corresponding elliptic points. Since the fundamental matrix is by definition holomorphic in the whole upper half-plane, in particular at the elliptic points, it follows that

(1) the matrices $\mathcal{A}$ and $\mathcal{B}$ are simple (i.e., diagonalizable), since otherwise $\boldsymbol{\Xi}(\tau)$ would have logarithmic singularities;

(2) the eigenvalues of $\mathcal{A}$ and $\mathcal{B}$ are non-negative to avoid poles;

(3) the eigenvalues of $\mathcal{A}$ and $\mathcal{B}$ are integers, otherwise $\boldsymbol{\Xi}(\tau)$ would have (algebraic or transcendental) branch points;

(4) no two eigenvalues of $\mathcal{A} / 2$ and $\mathcal{B} / 3$ may differ by non-zero integers, otherwise one would get logarithmic branch points.

These already restrict the matrices $\mathcal{A}$ and $\mathcal{B}$ to a great extent, but there is one more restriction, namely that all eigenvalues of $\mathcal{A} / 2$ and $\mathcal{B} / 3$ should be less than 1. This last condition is a completeness condition: would there be an eigenvalue greater or equal to one, the columns of the solution of Equation (3.3) would not span the full $\mathbb{C}[J]$-module $\mathcal{M}(\rho)$ (for the monodromy representation $\rho$ ). More precisely, let $P^{-1} A P$ be a diagonal matrix $D$, and suppose $D_{\eta \eta} \geq 2$; then the $\eta$ th column of $\boldsymbol{\Xi}(\mathfrak{z}) P$ will be a multiple of $\mathfrak{z}$. This column vector, as a function of $\tau$, could be divided by $J(\tau)-984$ while remaining holomorphic; the quotient would still be in $\mathcal{M}(\rho)$, but not in the $\mathbb{C}[J]$-span of the columns of $\boldsymbol{\Xi}(\tau)$. The argument for $\mathcal{B} / 3$ is similar, using $J(\tau)+744$ instead. 
This last completeness condition, together with the four analyticity conditions, imply the following.

Spectral condition: The possible eigenvalues of $\mathcal{A}$ are 0 or 1 , while those of $\mathcal{B}$ are either 0,1 or 2 .

This is a basic result, which restricts considerably the possible coefficient matrices. In particular, it implies that the characteristic polynomials of $\mathcal{A}$ and $\mathcal{B}$ read

$$
\begin{aligned}
\operatorname{det}(z-\mathcal{A}) & =z^{d-\alpha}(z-1)^{\alpha} \\
\operatorname{det}(z-\mathcal{B}) & =z^{d-\beta_{1}-\beta_{2}}(z-1)^{\beta_{1}}(z-2)^{\beta_{2}}
\end{aligned}
$$

where $d$ denotes their dimension, while the multiplicities $\alpha, \beta_{1}$ and $\beta_{2}$ are given by

$$
\begin{aligned}
\alpha & =\operatorname{Tr}(\mathcal{A}) \\
\beta_{1} & =2 \operatorname{Tr}(\mathcal{B})-\operatorname{Tr}\left(\mathcal{B}^{2}\right), \\
\beta_{2} & =\frac{1}{2}\left(\operatorname{Tr}\left(\mathcal{B}^{2}\right)-\operatorname{Tr}(\mathcal{B})\right) .
\end{aligned}
$$

The quadruple $\left(d, \alpha, \beta_{1}, \beta_{2}\right)$ of non-negative integers is a very important discrete invariant of the representation $\rho$, which we will call its signature. For example, the traces of the representation matrices

$$
S=\rho\left(\begin{array}{cc}
0 & -1 \\
1 & 0
\end{array}\right) \quad \text { and } \quad U=\rho\left(\begin{array}{cc}
0 & -1 \\
1 & -1
\end{array}\right)
$$

are completely determined by it $^{5}$ :

$$
\begin{aligned}
& \operatorname{Tr}(S)=d-2 \alpha \\
& \operatorname{Tr}(U)=d-\frac{3}{2}\left(\beta_{1}+\beta_{2}\right)+i \frac{\sqrt{3}}{2}\left(\beta_{1}-\beta_{2}\right) .
\end{aligned}
$$

We also note that

$$
\begin{aligned}
& \operatorname{Tr}(\mathcal{X})=4\left(62 \beta_{1}+124 \beta_{2}-123 \alpha\right) \\
& \operatorname{Tr}(\boldsymbol{\Lambda})=d-\frac{\alpha}{2}-\frac{\beta_{1}+2 \beta_{2}}{3}
\end{aligned}
$$

\footnotetext{
${ }^{5}$ Conversely, the traces of $S$ and $U$ - together with the dimension $d$ - determine the signature.
} 
In particular, the trace of the characteristic matrix $\mathcal{X}$ is always an integer divisible by 4 , which is congruent to $4 \alpha$ modulo 248 .

As another application of the notion of signature, let us mention the following formula for the determinant of the fundamental matrix:

$$
\operatorname{det} \boldsymbol{\Xi}(\tau)=\left(\frac{E_{4}(\tau)}{\Delta(\tau)^{1 / 3}}\right)^{\beta_{1}+2 \beta_{2}}\left(\frac{E_{6}(\tau)}{\Delta(\tau)^{1 / 2}}\right)^{\alpha}
$$

where $E_{4}$ and $E_{6}$ denote the (normalized) Eisenstein series of weights 4 and 6. The proof of this result is simple: since $\boldsymbol{\Xi}(\tau)$ satisfies Equation (2.14), its determinant satisfies - according to a theorem of Liouville — the differential equation

$$
\frac{1}{2 \pi i} \frac{d(\log \operatorname{det} \Xi(\tau))}{d \tau}=\operatorname{Tr} \mathfrak{D}(\tau)
$$

Moreover, it follows from Equation (2.16) that det $\boldsymbol{\Xi}(q)$ behaves as $q^{\operatorname{Tr}(\boldsymbol{\Lambda}-1)}$ for $q \rightarrow 0$. It is an easy matter to check that the RHS of Equation (3.11) satisfies the differential equation Equation (3.12) with this particular boundary condition, and by general theory such a solution is unique.

It follows in particular that the fundamental matrix is invertible everywhere except the elliptic points. That it can not be invertible at the elliptic points, for typical representations, is obvious: for example, at $\tau=i$ one has $\boldsymbol{\Xi}(i)=S \boldsymbol{\Xi}(i)$ because of Equation (2.25), and so $\boldsymbol{\Xi}(i)$ invertible would imply $S$ trivial.

Let us return to the spectral condition. It follows from Equation (3.5) that the minimal polynomials of $\mathcal{A}$ and $\mathcal{B}$ divide $z(z-1)$, respectively, $z(z-1)(z-2)$. Since any matrix is a root of its minimal polynomial, the spectral condition may be expressed as

$$
\mathcal{A}(\mathcal{A}-1)=\mathcal{B}(\mathcal{B}-1)(\mathcal{B}-2)=0
$$

Of the four matrices $\boldsymbol{\Lambda}, \mathcal{X}, \mathcal{A}$ and $\mathcal{B}$, any two determine the other two, ${ }^{6}$ e.g., Equations (3.4a) and (3.4b) imply that $\mathcal{B}=3(1-\boldsymbol{\Lambda}-\mathcal{A} / 2)$. Inserting this expression into Equation (3.13), one gets the following system of

\footnotetext{
${ }^{6}$ This is trivial unless two eigenvalues of $\boldsymbol{\Lambda}$ differ by 1 , but this can be always avoided by the use of transformations as in Equation (2.23).
} 
algebraic equations:

$$
\begin{aligned}
\mathcal{A}^{2}= & \mathcal{A} \\
\mathcal{A} \boldsymbol{\Lambda} \mathcal{A}= & -\frac{17}{18} \mathcal{A}-2\left(\mathcal{A} \boldsymbol{\Lambda}^{2}+\boldsymbol{\Lambda} \mathcal{A} \boldsymbol{\Lambda}+\boldsymbol{\Lambda}^{2} \mathcal{A}\right)+3(\mathcal{A} \boldsymbol{\Lambda}+\boldsymbol{\Lambda} \mathcal{A})-4 \boldsymbol{\Lambda}^{3} \\
& +8 \boldsymbol{\Lambda}^{2}-\frac{44}{9} \boldsymbol{\Lambda}+\frac{8}{9} .
\end{aligned}
$$

That is, for a given exponent matrix $\boldsymbol{\Lambda}$, the matrix $\mathcal{A}$ has to satisfy Equation (3.14): note that this is a simultaneous system of quadratic equations for the matrix elements of $\mathcal{A}$, and that the matrix $\boldsymbol{\Lambda}$ (which plays the role of a parameter) is diagonal. Once a solution to Equation (3.14) is known, the corresponding characteristic matrix may be determined from Equation (3.4a).

What can be said about the solutions of Equation (3.14)? First of all, if $(\boldsymbol{\Lambda}, \mathcal{X})$ is a solution and $M$ is a monomial matrix (i.e., the product of a diagonal and a permutation matrix), then $\left(M^{-1} \boldsymbol{\Lambda} M, M^{-1} \mathcal{X} M\right)$ is again a solution: more generally, this holds for any matrix $M$, provided that $M^{-1} \boldsymbol{\Lambda} M$ is still diagonal. These transformations do not change the equivalence class of the corresponding representation $\rho$, and may be used to put the solution into some useful standard form.

More interesting is duality, the involutive transformation $(\boldsymbol{\Lambda}, \mathcal{X}) \mapsto$ $\left(\boldsymbol{\Lambda}^{\vee}, \mathcal{X}^{\vee}\right)$ with $^{7}$

$$
\begin{aligned}
& \boldsymbol{\Lambda}^{\vee}=\frac{5}{6}-\boldsymbol{\Lambda}, \\
& \mathcal{X}^{\vee}=4-{ }^{t} \mathcal{X},
\end{aligned}
$$

which sends $\mathcal{A}$ to $\mathcal{A}^{\vee}=1-{ }^{t} \mathcal{A}$ and $\mathcal{B}$ to $\mathcal{B}^{\vee}=2-{ }^{t} \mathcal{B}$ : clearly, $\mathcal{A}^{\vee}$ and $\mathcal{B}^{\vee}$ satisfy the spectral condition if $\mathcal{A}$ and $\mathcal{B} \operatorname{did}$. The fundamental matrix corresponding to the dual pair $\left(\boldsymbol{\Lambda}^{\vee}, \mathcal{X}^{\vee}\right)$ is given by

$$
\boldsymbol{\Xi}^{\vee}(\tau)=\frac{E_{14}(\tau)}{\Delta^{7 / 6}(\tau)}\left({ }^{t} \boldsymbol{\Xi}(\tau)\right)^{-1}
$$

The prefactor is needed to ensure holomorphicity, which can be proved using Equation (3.11) and the spectral condition. The dual representation $\rho^{\vee}$ is equivalent to the tensor product of the contragredient of $\rho$ with the onedimensional representation $\varkappa$ appearing in the bottom row of table 1 below.

It is now time to establish the relation of invertibility of $\mathcal{P}$ to Equation (2.22), left pending in Section 2. If $\mathcal{P}$ is invertible, then a fundamental

\footnotetext{
${ }^{7}$ We denote by ${ }^{t} M$ the transpose of a matrix $M$.
} 
Table 1: One-dimensional representations.

\begin{tabular}{|c|c|c|c|c|c|c|c|c|}
\hline $\mathcal{A}$ & $\mathcal{B}$ & $\Lambda$ & $\mathcal{X}$ & $\boldsymbol{\Xi}(\tau)$ & $S$ & $T$ & $U$ & Name \\
\hline 0 & 0 & 1 & 0 & 1 & 1 & 1 & 1 & 1 \\
\hline 0 & 1 & $2 / 3$ & 248 & $\frac{E_{4}}{\Delta^{1 / 3}}=(J+744)^{1 / 3}$ & 1 & $\omega^{4}$ & $\omega^{2}$ & $\varkappa^{2}$ \\
\hline 0 & 2 & $1 / 3$ & 496 & $\frac{E_{8}}{\Delta^{2 / 3}}=(J+744)^{2 / 3}$ & 1 & $\omega^{2}$ & $\omega^{4}$ & $\varkappa^{4}$ \\
\hline 1 & 0 & $1 / 2$ & -492 & $\frac{E_{6}}{\Delta^{1 / 2}}=(J-984)^{1 / 2}$ & -1 & -1 & 1 & $\varkappa^{3}$ \\
\hline 1 & 1 & $1 / 6$ & -244 & $\frac{E_{10}}{\Delta^{5 / 6}}=(J+744)^{1 / 3}(J-984)^{1 / 2}$ & -1 & $\omega$ & $\omega^{2}$ & $\bar{\varkappa}$ \\
\hline 1 & 2 & $-1 / 6$ & 4 & $\frac{E_{14}}{\Delta^{7 / 6}}=(J+744)^{2 / 3}(J-984)^{1 / 2}$ & -1 & $\omega^{5}$ & $\omega^{4}$ & $\varkappa$ \\
\hline
\end{tabular}

matrix $\boldsymbol{\Xi}(\tau)$ satisfying Equation (2.16) exists for which the whole theory presented above holds. Comparing Equations (3.7),(3.8) and Equation (3.10), we arrive at Equation (2.22). In other words, while the integer part of $\boldsymbol{\Lambda}$ is to a great extent arbitrary, its trace is completely determined by the representation $\rho$.

More generally, given any $\mathbb{C}[J]$-submodule $M$ of $\mathcal{M}(\rho)$ of full rank $d$, linear algebra shows how to construct a matrix $\boldsymbol{\Xi}(\tau)$ of form Equation (2.13), for some choice of $\boldsymbol{\Lambda}$, such that $M$ is the $\mathbb{C}[J]$-span of the columns of $\boldsymbol{\Xi}(\tau)$. Moreover, $\operatorname{Tr}(\boldsymbol{\Lambda})$ will be bounded above by the RHS of Equation (2.22), with strict inequality if $M \neq \mathcal{M}(\rho)$ (to see this, use transformations like Equation (2.23) to make the $\boldsymbol{\Lambda} \mathrm{s}$ for $\mathcal{M}(\rho)$ and $M$ agree in all but one spot). If in addition the submodule is $\nabla$-stable, then that matrix $\boldsymbol{\Xi}(\tau)$ will satisfy 
Equation (3.3) for $\mathcal{A}, \mathcal{B}$ defined by Equations (3.4a) and (3.4b), although the eigenvalues of $\mathcal{A}$ and $\mathcal{B}$ can now be arbitrary non-negative integers. However, this submodule can be 'completed' using the method outlined in our proof of the spectral condition, by dividing the appropriate vectors by $J-984$ or $J+744$ (at each stage, the submodule will be $\nabla$-stable, owing to Equations (3.2),(3.3)). The result will be matrices $\boldsymbol{\Xi}(\tau), \boldsymbol{\Lambda}, \mathcal{A}, \mathcal{B}$ satisfying the spectral condition and Equation (2.22). To summarize, given a $\nabla$-stable rank $d$ submodule $M$ of $\mathcal{M}(\rho)$, with matrices $\boldsymbol{\Lambda}, \mathcal{A}, \mathcal{B}$, we have: $M=\mathcal{M}(\rho)$ iff $\boldsymbol{\Lambda}$ satisfies Equation (2.22), iff $\mathcal{A}, \mathcal{B}$ satisfy the spectral condition.

Those remarks permit an elementary and constructive proof of the invertibility of $\mathcal{P}$. It suffices to show that the $\mathbb{C}[J]$-module $\mathcal{M}(\rho)$ has rank $d$. That it cannot have rank greater than $d$ follows quickly from the fact that a non-constant function holomorphic on $\mathbf{H} / \mathrm{SL}_{2}(\mathbb{Z})$ must have poles at the cusps. It is enough then to find $d$ linearly independent vectors in $\mathcal{M}(\rho)$. Introduce the following notation: given a $q$-series $f(q)=q^{\ell} \sum_{n=0}^{\infty} a_{n} q^{n}$ with $a_{0} \neq 0$, define $\mathrm{o}(f)$ to be $\ell$, the order of the zero at $q=0-$ e.g., $\mathrm{o}(\eta)=$ $1 / 24$ and $\mathrm{o}(J)=-1$. The paper [11] explicitly constructs some weight $k$ vector-valued modular forms for $\rho$, namely the Poincaré series $P$, where $k$ here can be, e.g., any sufficiently large multiple of 12 . In particular, let $\mathbb{Y}^{(i)}(\tau)=P(\tau ; \rho, k, 1,-2, i)$ in their notation, for $1 \leq i \leq d$; then each $\mathbb{Y}^{(i)}$ is a vector-valued modular form for $\rho$ of weight $k$, holomorphic throughout $\mathbf{H}$, with o $\left(\mathbb{Y}_{i}^{(i)}\right)<0<\mathrm{o}\left(\mathbb{Y}_{j}^{(i)}\right)$ for all $j \neq i$. Thus each $\mathbb{X}^{(i)}=\mathbb{Y}^{(i)} / \Delta^{k / 12}$ lies in $\mathcal{M}(\rho)$; that they are all linearly independent over $\mathbb{C}(J)$ follows from the usual determinant argument.

\section{Low-dimensional examples}

This section is included to illustrate the effectiveness of the theory on some simple examples up to dimension 3. As we shall see, some of the non-trivial aspects of the theory already arise in these cases. As usual,

$$
S=\rho\left(\begin{array}{cc}
0 & -1 \\
1 & 0
\end{array}\right) \quad \text { and } \quad T=\rho\left(\begin{array}{ll}
1 & 1 \\
0 & 1
\end{array}\right)
$$

will denote the matrices representing the standard generators of $\mathrm{SL}_{2}(\mathbb{Z})$ and

$$
U=S T^{-1}=\rho\left(\begin{array}{ll}
0 & -1 \\
1 & -1
\end{array}\right)
$$

The first comment is that it is enough to consider indecomposable representations: indeed, if $\rho_{1}$ and $\rho_{2}$ are two representations of $\mathrm{SL}_{2}(\mathbb{Z})$ satisfying the criteria of Section 2, then their direct sum $\rho_{1} \oplus \rho_{2}$ also satisfies these 
criteria, and its exponent, characteristic and fundamental matrices are just the direct sums of the corresponding matrices of its summands:

$$
\begin{aligned}
& \boldsymbol{\Lambda}\left(\rho_{1} \oplus \rho_{2}\right)=\boldsymbol{\Lambda}\left(\rho_{1}\right) \oplus \boldsymbol{\Lambda}\left(\rho_{2}\right), \\
& \mathcal{X}\left(\rho_{1} \oplus \rho_{2}\right)=\mathcal{X}\left(\rho_{1}\right) \oplus \mathcal{X}\left(\rho_{2}\right), \\
& \boldsymbol{\Xi}\left(\rho_{1} \oplus \rho_{2}\right)=\mathbf{\Xi}\left(\rho_{1}\right) \oplus \mathbf{\Xi}\left(\rho_{2}\right) .
\end{aligned}
$$

Thus, in order to determine the above quantities for an arbitrary representation $\rho$, one should first decompose $\rho$ into a direct sum of indecomposable representations, and determine the relevant quantities for all the indecomposable constituents separately.

The representations of $\mathrm{SL}_{2}(\mathbb{Z})$ of dimension 1 that satisfy our criteria are easy to classify: in this case the representation matrices are mere numbers, and we get a total of six inequivalent representations, each of which is a tensor power of the representation $\varkappa$ defined in the last row of table 1 . Note that this is in complete accord with the spectral condition: there are exactly six pairs of $1 \times 1$ matrices that satisfy it. The corresponding exponent and characteristic matrices are easily determined, and this leads, via the compatibility equation Equation (2.14), to the corresponding fundamental matrices. ${ }^{8}$ The results are gathered in table 1 , where $\omega=\exp (2 \pi i / 6)$ and $E_{k}$ stands for the Eisenstein series of weight $k$.

The most interesting comments about table 1 are related to the first and last rows. In the first row we find the trivial representation, and one would naively expect that the corresponding exponent matrix is 0. But this choice does not satisfy Equation (2.22): we have to take $\boldsymbol{\Lambda}=1$ according to our definitions. And indeed, this choice is consistent with the fact that the constants belong to $\mathcal{M}(\rho)$ if $\rho$ is trivial. The last row is even more interesting: naively, one would take $\boldsymbol{\Lambda}=5 / 6$, but this would be again in conflict with Equation (2.22); the correct value is $\boldsymbol{\Lambda}=-1 / 6$. Indeed, if one would have $\Lambda=5 / 6$, then $\Delta^{1 / 6} \mathbb{X}^{(1 ; 1)}$ would be a weight 2 modular form for the trivial representation, but no such form exists, by classical arguments [1].

Let us now turn to higher dimensions. The pairs of matrices

$$
\begin{array}{cc}
\Lambda=\frac{1}{24}\left(\begin{array}{ll}
17 & \\
& 11
\end{array}\right), & \mathcal{X}=\left(\begin{array}{rr}
133 & 1248 \\
56 & -377
\end{array}\right), \\
\Lambda=\frac{1}{24}\left(\begin{array}{ll}
23 & \\
& 5
\end{array}\right), \quad \mathcal{X}=\left(\begin{array}{rr}
3 & 26752 \\
2 & -247
\end{array}\right),
\end{array}
$$

\footnotetext{
${ }^{8}$ There is no need to solve the differential equation in this case: the fundamental matrices can be determined by purely function theoretic arguments, or even better, from the determinantal formula Equation(3.11).
} 
both correspond to dimension 2 representations $\rho$ with the same matrix

$$
S=\frac{1}{\sqrt{2}}\left(\begin{array}{rr}
1 & 1 \\
1 & -1
\end{array}\right)
$$

(of course, $T=\exp (2 \pi i \boldsymbol{\Lambda})$ by definition). The corresponding fundamental matrices have $q$-expansions

$$
q^{\Lambda}\left(\begin{array}{cc}
q^{-1}+133+1673 q+11914 q^{2}+\cdots & 1248+49504 q+806752 q^{2}+\cdots \\
56+968 q+7504 q^{2}+\cdots & q^{-1}-377-22126 q-422123 q^{2}-\cdots
\end{array}\right)
$$

and

$$
q^{\Lambda}\left(\begin{array}{cc}
q^{-1}+3+4 q+7 q^{2}+\cdots & 26752+1734016 q+46091264 q^{2}+\cdots \\
2+2 q+6 q^{2}+\cdots & q^{-1}-247-86241 q-4182736 q^{2}-\cdots
\end{array}\right) .
$$

They describe representations associated to the Wess-Zumino-NovikovWitten models [6] of level 1 based on the Lie algebras $E_{7}$ and $A_{1}$, respectively (whose dimensions 133 and 3 appear as $\mathcal{X}_{11}$, and whose character vectors are given by the first columns of the corresponding fundamental matrix).

More generally, the solution for an arbitrary two-dimensional $\mathrm{SL}_{2}(\mathbb{Z})$ representation $\rho$ can be obtained in closed form - for example, the fundamental matrices can be expressed as linear combinations of classical hypergeometric series.

In dimension 3 , the sequence

$$
\begin{aligned}
\boldsymbol{\Lambda}_{k} & =\frac{1}{48}\left(\begin{array}{ccc}
47-2 k & \\
& 23-2 k & \\
& 2+4 k
\end{array}\right), \\
\mathcal{X}_{k} & =\left(\begin{array}{ccc}
k(2 k+1) & \frac{1}{3}(31-2 k)(9+2 k)(25+2 k) & 2^{12-k}(23-2 k) \\
2 k+1 & (11-k)(25+2 k) & -2^{12-k} \\
2^{k} & -2^{k}(25+2 k) & 2 k-23
\end{array}\right),
\end{aligned}
$$

where $k$ is an integer in the range $0 \leq k<12$, corresponds to representations that share the same matrix

$$
S=\frac{1}{2}\left(\begin{array}{ccc}
1 & 1 & \sqrt{2} \\
1 & 1 & -\sqrt{2} \\
\sqrt{2} & -\sqrt{2} & 0
\end{array}\right) .
$$

For $k=0$ one recovers the representation associated to the Ising model [6]: in this case, the fundamental matrix may be expressed in terms of Weber functions [4]. 
What happens in these examples holds more generally: different PSLrepresentations can have identical matrix $S$, but (in dimension $\leq 5$ [16], though not higher) an irreducible representation is completely determined by $T$. (To see this, recall that [16] proves that, in dimension $\leq 5$, an irreducible braid group $B_{3}$ representation is uniquely determined by the eigenvalues of a matrix $A$ and a parameter $\delta$; but PSL is a quotient of $B_{3}$, and its representations correspond to $\delta=1$ and $T=A$.)

One striking feature of all the above examples is that their characteristic matrices are all integral (in a suitable basis). This is far from being trivial, since most solutions of Equation (3.14) have irrational $\mathcal{X}$. Actually, the reason for using the pair $(\boldsymbol{\Lambda}, \mathcal{X})$ to characterize the representation $\rho$, instead of e.g., the pair $(\mathcal{A}, \mathcal{B})$, comes from the observation that for representations $\rho$ which have a conformal field theory origin, there always seems to exist a basis in which the characteristic matrix is integral. We will explore this issue in Section 6.

\section{The inversion formula}

We have seen above that the knowledge of the fundamental matrix $\boldsymbol{\Xi}(\tau)$ allows the determination of all canonical basis vectors through solving the recursion relations, and this in turn allows to determine the unique element $\mathbb{X} \in \mathcal{M}(\rho)$ with a given principal part $\mathcal{P} \mathbb{X}$. Actually, there exists an explicit inversion formula which gives $\mathbb{X}$ in terms of $\mathcal{P} \mathbb{X}$ and the fundamental matrix.

Inversion formula: For $\mathbb{X}(q) \in \mathcal{M}(\rho)$ with principal part $\mathcal{P} \mathbb{X}$, one has

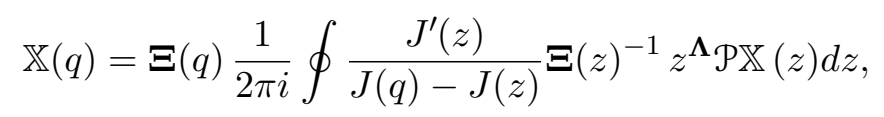

where $J^{\prime}(z)=-z^{-2}+\sum_{n=1}^{\infty} n c(n) z^{n-1}$ is the derivative of $J$, and the integral is over a closed contour encircling the origin and contained in the circle of radius $|q|$.

Proof. Since the principal part map $\mathcal{P}$ is linear, it is enough to prove Equation (5.1) for the canonical basis vectors, in which case it reads

$$
\left[\mathbb{X}^{(\xi ; n)}(q)\right]_{\eta}=\frac{1}{2 \pi i} \oint \frac{z^{\boldsymbol{\Lambda}_{\xi \xi}-n} J^{\prime}(z)}{J(q)-J(z)}\left[\boldsymbol{\Xi}(q) \boldsymbol{\Xi}(z)^{-1}\right]_{\eta \xi} d z
$$


To see that Equation (5.2) holds, let us introduce the matrix valued generating function

$$
\mathfrak{X}_{\xi \eta}(q, z)=\sum_{n=1}^{\infty}\left[\mathbb{X}^{(\eta ; n)}(q)\right]_{\xi} z^{n-1}
$$

As we will see below, this series has a non-zero radius of convergence around $z=0$, and thus defines a holomorphic function of $z$ in a small enough neighborhood, for any fixed value of $q$. This means that $z^{-n} \mathfrak{X}_{\xi \eta}(q, z)$ has a pole at $z=0$ whose residue is

$$
\left[\mathbb{X}^{(\eta ; n)}(q)\right]_{\xi}=\frac{1}{2 \pi i} \oint z^{-n} \mathfrak{X}_{\xi \eta}(q, z) d z,
$$

by the residue theorem.

Multiplying both sides of the recursion relation Equation (2.7) by $z^{m}$, and summing from $m=1$, one gets

$$
\begin{aligned}
\mathfrak{X}_{\xi \eta}(q, z)- & \boldsymbol{\Xi}(q)_{\xi \eta}=z J(q) \mathfrak{X}_{\xi \eta}(q, z) \\
& -\sum_{m=1}^{\infty} \sum_{n=1}^{m-1} c(n)\left[\mathbb{X}^{(\eta ; m-n)}(q)\right]_{\xi} z^{m}-\sum_{\rho} \mathcal{X}_{\rho \eta}(z) \boldsymbol{\Xi}(q)_{\xi \rho},
\end{aligned}
$$

where

$$
\mathcal{X}_{\xi \eta}(z)=\sum_{m=1}^{\infty} \mathcal{X}_{\xi}^{(\eta ; m)} z^{m}
$$

The double sum on the RHS of Equation (5.5) may be rearranged as follows:

$$
\begin{gathered}
\sum_{m=1}^{\infty} \sum_{n=1}^{m-1} c(n)\left[\mathbb{X}^{(\eta ; m-n)}(q)\right]_{\xi} z^{m}=\sum_{n=1}^{\infty} \sum_{m=n+1}^{\infty} c(n) z^{n}\left[\mathbb{X}^{(\eta ; m-n)}(q)\right]_{\xi} z^{m-n} \\
=\sum_{n=1}^{\infty} \sum_{k=1}^{\infty} c(n) z^{n}\left[\mathbb{X}^{(\eta ; k)}(q)\right]_{\xi} z^{k}=z\left(J(z)-z^{-1}\right) \mathfrak{X}_{\xi \eta}(q, z),
\end{gathered}
$$

so that finally Equation (5.5) reads

$$
z(J(q)-J(z)) \mathfrak{X}(q, z)=\boldsymbol{\Xi}(q)(\mathcal{X}(z)-\mathbf{1}) .
$$

We still have to determine the generating function $\mathcal{X}(z)$. To do this, let us consider Equation (5.7) in the limit when $q$ approaches $z$ : on the RHS we 
get simply $\boldsymbol{\Xi}(z)(\mathcal{X}(z)-\mathbf{1})$, while on the LHS all terms vanish because of the factor $(J(q)-J(z))$, except for those that are singular in $q$, which yield

$$
\begin{aligned}
& \lim _{q \rightarrow z}\left\{z(J(q)-J(z)) q^{\boldsymbol{\Lambda}} \sum_{m=1}^{\infty} q^{-m} z^{m-1}\right\}= \\
& z^{\boldsymbol{\Lambda}+1} \lim _{q \rightarrow z}\left\{\frac{J(q)-J(z)}{q-z}\right\}=z^{\boldsymbol{\Lambda}+1} J^{\prime}(z) .
\end{aligned}
$$

Note that the geometric sum is convergent for $|z|<|q|$. All in all, we get

$$
\mathcal{X}(z)-\mathbf{1}=J^{\prime}(z) \boldsymbol{\Xi}(z)^{-1} z^{\mathbf{1}+\boldsymbol{\Lambda}}
$$

Inserting this last expression into Equation (5.7), we arrive at

$$
\mathfrak{X}(q, z)=\frac{J^{\prime}(z)}{J(q)-J(z)} \boldsymbol{\Xi}(q) \boldsymbol{\Xi}(z)^{-1} z^{\mathbf{\Lambda}},
$$

and this - together with Equation (5.4) — leads to the inversion formula. Since the fundamental matrix is invertible except for the elliptic points, Equation (5.9) shows that the generating function $\mathfrak{X}_{\xi \eta}(q, z)$ is indeed convergent for small enough $|z|<|q|$.

Let us stress that the above proof gives more than just the inversion formula: it provides closed expressions for all the canonical basis vectors, as well as for their generating function $\mathfrak{X}(q, z)$. Incidentally, in the case of the trivial representation Equation (5.9) is related to the "bivarial transformation" [13] of Monstrous Moonshine.

We can now substantiate our claim in footnote 3 , namely that the differential equation Equation (2.11) satisfied by the fundamental matrix is enough to guarantee the compatibility of the recursion and differential relations. Indeed, multiplying both sides of Equation (2.11) by $z^{m-1}$ and summing over $m$, we get on the RHS

$$
\sum_{m=1}^{\infty}\left[\nabla \mathbb{X}^{(\eta ; m)}(q)\right]_{\xi} z^{m-1}=q \mathcal{E}(q) \frac{\partial}{\partial q} \mathfrak{X}_{\xi \eta}(q, z)
$$


while on the LHS one has

$$
\begin{aligned}
\sum_{m=1}^{\infty} \sum_{\rho} \boldsymbol{\Lambda}_{\rho \rho} \mathcal{X}_{\rho}^{(\eta ; m)} & \Xi(q)_{\xi \rho} z^{m-1} \\
& +\sum_{m=1}^{\infty} \sum_{n=-1}^{m-1}\left(\boldsymbol{\Lambda}_{\eta \eta}-m\right) \mathcal{E}_{n}\left[\mathbb{X}^{(\eta ; m-n)}(q)\right]_{\xi} z^{m-1} .
\end{aligned}
$$

The first term in Equation (5.11) gives simply

$$
z^{-1}[\boldsymbol{\Xi}(q) \boldsymbol{\Lambda} \mathcal{X}(z)]_{\xi \eta},
$$

while the double sum in the second term may be rearranged as follows

$$
\begin{gathered}
\sum_{n=-1}^{\infty} \sum_{m=n+1}^{\infty} \mathcal{E}_{n}\left[\mathbb{X}^{(\eta ; m-n)}(q)\right]_{\xi}\left(\boldsymbol{\Lambda}_{\eta \eta}-m\right) z^{m-1}-z^{-1} \boldsymbol{\Lambda}_{\eta \eta}\left[\mathbb{X}^{(\eta ; 1)}(q)\right]_{\xi} \\
=\sum_{n=-1}^{\infty} \sum_{m=n+1}^{\infty} \mathcal{E}_{n}\left[\mathbb{X}^{(\eta ; m-n)}(q)\right]_{\xi}\left(\frac{\boldsymbol{\Lambda}_{\eta \eta}}{z}-\frac{\partial}{\partial z}\right) z^{m}-z^{-1} \boldsymbol{\Lambda}_{\eta \eta}\left[\mathbb{X}^{(\eta ; 1)}(q)\right]_{\xi} \\
=\left(\frac{\boldsymbol{\Lambda}_{\eta \eta}}{z}-\frac{\partial}{\partial z}\right)\left\{z \mathcal{E}(z) \mathfrak{X}_{\xi \eta}(q, z)\right\}-z^{-1} \boldsymbol{\Lambda}_{\eta \eta} \boldsymbol{\Xi}(q)_{\xi \eta}
\end{gathered}
$$

All in all, we arrive at the conclusion that the differential relations Equation (2.11) are equivalent to the following partial differential equation for the generating function $\mathfrak{X}(q, z)$ :

$$
q \mathcal{E}(q) \frac{\partial}{\partial q} \mathfrak{X}(q, z)+\frac{\partial}{\partial z}\{z \mathcal{E}(z) \mathfrak{X}(q, z)\}=\mathcal{E}(z) \mathfrak{X}(q, z) \boldsymbol{\Lambda}+\boldsymbol{\Xi}(q) \boldsymbol{\Lambda} \frac{\mathcal{X}(z)-\mathbf{1}}{z}
$$

It is now straightforward to show, using the relation (cf. Equation (3.2))

$$
q \mathcal{E}(q) J^{\prime}(q)=(J(q)+744)(984-J(q))
$$

that $\mathfrak{X}(q, z)$ - as given by Equation (5.9) — satisfies Equation (5.13), provided $\boldsymbol{\Xi}(q)$ satisfies the compatibility equation Equation (2.14).

\section{Positivity and integrality}

The representations $\rho$ of most interest to us (coming from conformal field theories and vertex operator algebras) have character vectors $\mathbb{X} \in \mathcal{M}(\rho)$ 
which are dimensions of $\mathbb{Z}$-graded vector spaces, and so their $q$-expansions Equation (2.3) have non-negative integer coefficients $\mathbb{X}[n]$. In this section, we find conditions on $\rho$ for the existence of such $\mathbb{X}$. Incidentally, this is also why we choose $\boldsymbol{\Lambda}$ and $\mathcal{X}$ for our fundamental data: in the cases of most interest to us, $\mathcal{X}$ is integral.

Throughout this section, let $\rho$ be an indecomposable matrix representation of $\mathrm{PSL}_{2}(\mathbb{Z})$ such that $T$ is diagonal and unitary. Call a non-zero vector $\mathbb{X}$ non-negative (resp. integral) if all its $q$-coefficients are non-negative real numbers (resp. integral). Recall the map o $\left(\sum_{n \geq 0} a_{n} q^{n+\ell}\right)=\ell$ of Section 3 . First, we give some easy conditions for non-negativity.

Non-negativity test: Suppose $\rho$ has a non-negative $\mathbb{X} \in \mathcal{M}(\rho)$. Then the matrix $S$ must have a strictly positive eigenvector with eigenvalue 1. Suppose in addition there is a unique component of $\mathbb{X}$, call it $\mathbb{X}_{0}(\tau)$, with a pole at $q=0$ of maximal order: i.e., $\mathrm{o}\left(\mathbb{X}_{0}\right)<\mathrm{o}\left(\mathbb{X}_{\nu}\right)$ for all $\nu \neq 0$. Then every entry in the 0 th column of $S$ must be a non-negative real number.

This uniqueness assumption holds, e.g., for any canonical basis vector; it also holds for the character vector $\mathbb{X}$ coming from a (unitary) conformal field theory, where it corresponds to the vacuum primary field.

The proof is easy. The eigenvector will be the vector $\mathbb{X}(\tau)$ evaluated at $\tau=i$, i.e., $q=e^{-2 \pi}$ : it is positive because $q>0$, and it has eigenvalue 1 because $\tau \mapsto-1 / \tau$ fixes $i$. Next, choose any $\eta$ such that $S_{\eta 0} \neq 0$; as $\tau$ approaches 0 along the imaginary axis, the component $\mathbb{X}_{\eta}(\tau)$ remains manifestly positive. Applying $\tau \mapsto-1 / \tau$, this is equivalent to $\tau$ approaching $i \infty$ along the imaginary axis (i.e., $q \rightarrow 0$ ), of $\sum_{\mu} S_{\eta \mu} \mathbb{X}_{\mu}(\tau)$. But by the uniqueness hypothesis, this is dominated by the $\mu=0$ term. Hence positivity forces $S_{\eta 0} \geq 0$ for that $\eta$.

Most $\rho$ fail the first condition: e.g., measure-0 of two-dimensional and four-dimensional representations, and 1/8th of three-dimensional ones, satisfy it. The second condition is more powerful: e.g., it quickly shows that any central charge $c<24$ conformal field theories or vertex operator algebras with modular representation identical to that of the Ising model, will have character vectors identical to it. More generally, it implies that there will be only finitely many possibilities for the character vectors of $c<24$ theories, with fixed modular representation.

Now let us turn to integrality. As we will now explain, the existence of integral $\mathbb{X}$ leads us directly to representations $\rho$ whose kernel is a congruence subgroup, i.e., $\operatorname{ker} \rho$ contains some principal congruence group

$$
\Gamma(N)=\left\{A \in \mathrm{SL}_{2}(\mathbb{Z}) \mid A \equiv 1(\bmod N)\right\}
$$


Each component $\mathbb{X}_{\eta}(\tau)$ of $\mathbb{X} \in \mathcal{M}(\rho)$ will be a modular function for the kernel ker $\rho$, which we will require here to be of finite index in $\mathrm{SL}_{2}(\mathbb{Z})$. Most such subgroups are non-congruence. An example of a modular function for a non-congruence subgroup is

$$
\sqrt{\frac{\eta(\tau)}{\eta(13 \tau)}}=q^{-1 / 4}\left(1-\frac{1}{2} q-\frac{5}{8} q^{2}-\frac{5}{16} q^{3}-\frac{45}{128} q^{4}+\cdots\right) .
$$

Although its Fourier coefficients are all rational, they have unbounded denominator. Indeed, the following observation is due originally to Atkin and Swinnerton-Dyer [2]:

Integrality conjecture: Suppose $f(\tau)=q^{c} \sum_{n=0}^{\infty} a_{n} q^{n / b}$ is a modular function, holomorphic in $\mathbf{H}$, for some subgroup $G$ of $\mathrm{SL}_{2}(\mathbb{Z})$ with finite index, where $c$ is rational and $b$ is a positive integer. If all coefficients $a_{n}$ are algebraic integers, then $G$ is a congruence subgroup.

Conversely, a modular function $f$ for $\Gamma(N)$ has a $q$-expansion of the form

$$
f(\tau)=\sum_{n=-\infty}^{\infty} a_{n} q^{n / N}
$$

where $a_{n}=0$ for all but finitely many $n<0$; if $f$ is holomorphic in $\mathbf{H}$, the denominators of its coefficients $a_{n}$ (if rational) will be bounded. The integrality conjecture implies that $\rho$ can have integral $\mathbb{X}$ only if $\operatorname{ker} \rho$ is congruence. That the kernel is a congruence subgroup for a representation coming from rational conformal field theory was established in [3].

Suppose for the remainder of this section that the kernel of $\rho$ contains some $\Gamma(N)$ - in that case $N$ can be taken to be the order of $T$. This implies that $\rho$ can equivalently be interpreted as a representation of the finite group $\mathrm{SL}_{2}\left(\mathbb{Z}_{N}\right)$, where $\mathbb{Z}_{N}=\mathbb{Z} / N \mathbb{Z}$. Incidentally, this congruence subgroup hypothesis is straightforward to verify for any given $\rho$, using the presentations of $\mathrm{SL}_{2}(\mathbb{Z}[1 / p])$ in $[9]$, but in practise a very convenient test is that if ker $\rho$ is a congruence subgroup, then for all integers $\ell$ coprime to $N$, the diagonal entries of $T^{\ell^{2}}$ and $T$ are identical apart from order. To see this, let

$$
G_{\ell}=S T^{1 / \ell} S T^{\ell} S T^{1 / \ell}=\rho\left(\begin{array}{cc}
\ell & 0 \\
0 & \ell^{-1}
\end{array}\right),
$$

where $1 / \ell$ is the inverse of $\ell \bmod N$; then $G_{\ell} T G_{\ell}^{-1}=T^{\ell^{2}}$.

Now, any finite-dimensional representation of a finite group is equivalent to one defined over some cyclotomic field $\mathbb{Q}_{L}=\mathbb{Q}\left[\xi_{L}\right]$, where $\xi_{L}$ is the root 
of unity $e^{2 \pi i / L}$. Replacing $N$ if necessary by multiple, thus we can (and will) assume that $\rho$ is a representation of $\mathrm{SL}_{2}\left(\mathbb{Z}_{N}\right)$, and all entries of all matrices $\rho(\gamma)$ lie in $\mathbb{Q}_{N}$. Call any such $\rho$ " $N$-defined". Call $\mathbb{X}$ rational (resp. $\mathbb{Q}_{N}$-rational) if all coefficients in the $q$-expansions of each component $\mathbb{X}_{\eta}(\tau)$ are rational numbers (resp. in $\mathbb{Q}_{N}$ ). It is known that if $\mathbb{X}$ is rational and ker $\rho$ is congruence, then some non-zero multiple $n \mathbb{X}$ will be integral. The remainder of this section is devoted to stating and proving a necessary and sufficient condition for rationality. Not surprisingly this involves the language of Galois.

For any $\ell$ coprime to $N$, let $\sigma_{\ell} \in \operatorname{Gal}\left(\mathbb{Q}_{N} / \mathbb{Q}\right)$ be the Galois automorphism sending $\xi_{N}$ to $\xi_{N}^{\ell}$. Let $\sigma_{\ell}$ act on any matrix $A \in M_{d \times d}\left(\mathbb{Q}_{N}\right)$ entry-wise.

Rationality test: Let $\rho$ be $N$-defined, and $\mathbb{X} \in \mathcal{M}(\rho)$ have components $\mathbb{X}_{\eta}$ whose coefficients $a_{\eta, n}, n \leq 0$, in Equation (6.3) are all rational. Then $\mathbb{X}$ is rational (hence a multiple is integral) iff for all $\ell$ coprime to $N$,

$$
\sigma_{\ell}(S)=G_{\ell} S
$$

where $G_{\ell}$ is defined in Equation (6.4). In this case, $G_{\ell}$ is a $\mathbb{Q}$-matrix and $S$ is real, and every column of $\boldsymbol{\Xi}(\tau)$ is rational.

The starting point for proving this is the observation that any component $\mathbb{X}_{\eta}(\tau)$ of any vector $\mathbb{X} \in \mathcal{M}(\rho)$ is among other things a modular function for $\Gamma(N)$. The theory of these functions is quite rich (see, e.g., [15, Chapter 6$]$ or $[12$, Chapter 6]).

Note that any $\mathbb{X} \in \mathcal{M}(\rho)$ is $\mathbb{Q}_{N}$-rational iff all coefficients in the principal part are in $\mathbb{Q}_{N}$. In particular, every canonical basis vector $\mathbb{X}^{(\eta ; m)}$ is $\mathbb{Q}_{N^{-}}$ rational. The reason for this is that the space of modular forms for $\Gamma(N)$ of any weight $k$ has a basis with integral $q$-expansions, so does the space of modular functions for $\Gamma(N)$, holomorphic in $\mathbf{H}$ and with bounded poles at the cusps; we can express $\mathbb{X}_{\eta}(\tau)$ in terms of these basis functions by matching behaviors at the cusps, and because $\rho$ is $N$-defined the coefficients will never leave the field $\mathbb{Q}_{N}$.

These Galois automorphisms $\sigma_{\ell}$ act on the data $(\Lambda, \mathcal{X}, \mathcal{A}, \mathcal{B}, \boldsymbol{\Xi}(\tau), \rho)$ associated to any $N$-defined $\rho$, as follows. Note that the matrices in Equations (3.4a,b) corresponding to $\Lambda$ and $\sigma_{\ell} \mathcal{X}$ will be $\sigma_{\ell} \mathcal{A}$ and $\sigma_{\ell} \mathcal{B}$, and thus the spectral condition will be satisfied - indeed the signature $\left(d, \alpha, \beta_{1}, \beta_{2}\right)$ would not have changed. It is easy to verify that the differential equation Equation (3.3) will have solution $\sigma_{\ell} \boldsymbol{\Xi}(\tau)$, where we apply $\sigma_{\ell}$ entry-by-entry, and its action on a $\mathbb{Q}_{N}$-rational $q$-series Equation (6.3) 
is simply

$$
\left(\sigma_{\ell} f\right)(\tau)=\sum_{n=-\infty}^{\infty} \sigma_{\ell}\left(a_{n}\right) q^{n / N} .
$$

By the above series, these $q$-series will be holomorphic throughout $\mathbf{H}$. The corresponding $\mathrm{PSL}_{2}(\mathbb{Z})$-representation $\tilde{\rho}$ can be found by the following consideration.

Let $\mathcal{H}_{N}$ be the modular functions $f$ for $\Gamma(N)$, holomorphic throughout $\mathbf{H}$, with coefficients $a_{n} \in \mathbb{Q}_{N}$. The group $\mathrm{GL}_{2}\left(\mathbb{Z}_{N}\right)$ acts on $\mathcal{H}_{N}$ on the right, i.e., $\left.f\right|_{\alpha \circ \beta}=\left.\left(\left.f\right|_{\alpha}\right)\right|_{\beta}$, as follows (see [12, Section 6.3] for more details). $\mathrm{GL}_{2}\left(\mathbb{Z}_{N}\right)$ is generated by $\mathrm{SL}_{2}\left(\mathbb{Z}_{N}\right)$, together with all matrices of the form $M_{\ell}=\left(\begin{array}{ll}1 & 0 \\ 0 & \ell\end{array}\right)$ where $\ell$ is coprime to $N . \quad \gamma \in \mathrm{SL}_{2}\left(\mathbb{Z}_{N}\right)$ acts on $\mathcal{H}_{N}$ in the obvious way: first lift to $\mathrm{SL}_{2}(\mathbb{Z})$, then act on $\tau$ by that fractional linear transformation. Moreover, $\left.f\right|_{M_{\ell}}=\sigma_{\ell} f$, as given by Equation (6.6), recovering the action on $\boldsymbol{\Xi}(\tau)$ we obtained last paragraph. That $\sigma_{\ell} f$ is holomorphic in $\mathbf{H}$ iff $f$ follows from the previous paragraph (though this is presumably also known classically). Then, writing $A=\left(\begin{array}{ll}a & b \\ c & d\end{array}\right)$, we have the calculation

$$
\begin{aligned}
& \left(\sigma_{\ell} \mathbb{X}\right)\left(\frac{a \tau+b}{c \tau+d}\right)=\left.\mathbb{X}\right|_{M_{\ell} A}(\tau)=\sigma_{\ell}\left(\left.\mathbb{X}\right|_{M_{\ell} A M_{\ell}^{-1}}\right)(\tau) \\
& \quad=\sigma_{\ell}\left(\rho\left(\begin{array}{cc}
a & \ell^{-1} b \\
\ell c & d
\end{array}\right) \mathbb{X}(\tau)\right)=\left(\sigma_{\ell} \rho\left(\begin{array}{cc}
a & \ell^{-1} b \\
\ell c & d
\end{array}\right)\right) \sigma_{\ell} \mathbb{X}(\tau)
\end{aligned}
$$

where $\ell^{-1}$ denotes the inverse of $\ell \bmod N$. Hence we obtain

$$
\tilde{\rho}\left(\begin{array}{ll}
a & b \\
c & d
\end{array}\right)=\sigma_{\ell}\left(\rho\left(\begin{array}{cc}
a & \ell^{-1} b \\
\ell c & d
\end{array}\right)\right) .
$$

More generally, if $\mathbb{X} \in \mathcal{M}(\rho)$ is $\mathbb{Q}_{N}$-rational, then the same argument shows that $\sigma_{\ell} \mathbb{X}$ lies in $\mathcal{M}(\tilde{\rho})$.

To complete the proof of the rationality test, note that for all $\ell$ coprime to $N, \sigma_{\ell} \mathbb{X} \in \mathcal{M}(\rho)$ iff

$$
\rho\left(\begin{array}{ll}
a & b \\
c & d
\end{array}\right)=\sigma_{\ell}\left(\rho\left(\begin{array}{cc}
a & \ell^{-1} b \\
\ell c & d
\end{array}\right)\right) .
$$

Each component $\left(\sigma_{\ell} \mathbb{X}\right)_{\eta}$ will have the same coefficients $a_{n}$ as $\mathbb{X}_{\eta}$, for all $n \leq 0$, and will be holomorphic in $\mathbf{H}$. Hence $\sigma_{\ell} \mathbb{X}=\mathbb{X}$ for all $\ell$, i.e., $\mathbb{X}$ is rational. Now, it suffices to test condition Equation (6.8) at the generators $S$ and $T$. One leads to Equation (6.5), and the other to $T=\sigma_{\ell} T^{\ell^{-1}}$, which 
is automatically satisfied. That $S$ is real follows from complex conjugation $\ell=-1$ in Equation (6.5). That $G_{\ell}$ is rational follows from the calculation

$$
G_{\ell^{\prime}} G_{\ell} S=\sigma_{\ell^{\prime} \ell} S=\left(\sigma_{\ell^{\prime}} G_{\ell}\right) G_{\ell^{\prime}} S=\sigma_{\ell^{\prime}}\left(G_{\ell} G_{\ell^{\prime}}\right) S=\sigma_{\ell^{\prime}}\left(G_{\ell^{\prime}} G_{\ell}\right) S
$$

The condition Equation (6.5) is automatic in conformal field theory in this case $G_{\ell}$ is in fact monomial.

\section{Summary and outlook}

This paper solves the Riemann-Hilbert problem for $\operatorname{PSL}_{2}(\mathbb{Z})$ : given a representation $\rho$, we have a differential equation Equation (3.3) whose monodromy is determined by $\rho$. The solution of this differential equation is the fundamental matrix $\boldsymbol{\Xi}(\tau)$ of Equation (2.13) — given it, any vectorvalued modular function $\mathbb{X}$ with multiplier $\rho$ can be uniquely determined from the inversion formula Equation (5.1). As an application of this theory, explicit bases for - and dimensions of - spaces of vector-valued modular forms of half-integer weight can be found. In practice, the most interesting vector-valued modular functions have non-negative integer $q$-expansions; the consequences for $\rho$ of the existence of such vectors is worked out in Section 6.

A number of future developments are suggested by the analysis of this paper. It is tempting to guess that the theory developed here can be extended to other genus-0 discrete subgroups of $\mathrm{PSL}_{2}(\mathbb{R})$. There are 6486 such groups with the additional property that they contain some $\Gamma(N)[5]$ : roughly a third of these have only one cusp - these may be the ones most accessible to our methods.

Vector-valued modular forms of arbitrary weight can be reduced to the modular functions studied here, by dividing by an appropriate power of $\eta(\tau)$. Such modular forms arise naturally in conformal field theory, and so this extension should be pursued. Knopp and Mason [11] have addressed questions like the asymptotic growth of Fourier coefficients of these modular forms, with methods apparently more effective when the weight is higher. Our results would complement theirs: we would obtain bases and dimensions for any weight.

One could also speculate about the possibility of considering infinitedimensional representations of $\mathrm{SL}_{2}(\mathbb{Z})$, which appear, for instance, in quasirational conformal field theory. In this case an indirect approach could prove fruitful: first, solve Equation (3.14) in an arbitrary Banach algebra, 
then consider the solutions of the corresponding differential equation Equation (3.3); of course, all relevant quantities that make sense will take their value in the given Banach algebra. The technicalities involved are far from being clear.

Integrality and positivity, already touched upon in Section 6, lead to many deep questions. For example, not all choices of $\boldsymbol{\Lambda}$ compatible with the trace formula Equation (2.22) are equally good: integrality, for instance, can be gained or lost by transformations as in Equation (2.24), as Equation (2.23) shows. It would be interesting to understand better how to choose the most suitable $\boldsymbol{\Lambda}$ in this respect.

\section{Acknowledgments}

The work of P.B. was supported by grants OTKA T047041, T043582, the János Bolyai Research Scholarship of the Hungarian Academy of Sciences and EC Marie Curie MRTN-CT-2004-512194. T.G. would like to thank Eötvös University and the University of Hamburg for kind hospitality while this research was undertaken; his research is supported in part by NSERC and the Humboldt Foundation.

\section{Appendix. The reduction of the modular representation}

To any rational CFT is associated a finite-dimensional representation $\varrho$ of $\mathrm{SL}_{2}(\mathbb{Z})$, where in general $\varrho\left(\begin{array}{cc}-1 & 0 \\ 0 & -1\end{array}\right)$ is not the identity, but a permutation matrix (charge conjugation). Nevertheless, exploiting the fact that characters of charge conjugate primaries are equal, one can associate to such a $\varrho$ a representation $\rho$ for which $\rho\left(\begin{array}{cc}-1 & 0 \\ 0 & -1\end{array}\right)$ is the unit matrix, so that the results of the paper may be applied. As far as conformal characters are concerned, it is only $\rho$ that matters.

The procedure is as follows: let

$$
T=\varrho\left(\begin{array}{ll}
1 & 1 \\
0 & 1
\end{array}\right) \quad \text { and } \quad S=\varrho\left(\begin{array}{cc}
0 & -1 \\
1 & 0
\end{array}\right)
$$

as usual. We know that $S^{2}$ is a permutation matrix of order 2 , representing charge conjugation. An orbit $\eta$ of charge conjugation has either length $|\eta|=1$ or length $|\eta|=2$. For any such orbit $\eta$ we select a representative $\eta^{*} \in \eta$. 
Define matrices $\mathcal{T}$ and $\mathcal{S}$, whose rows and columns are indexed by these orbits $\eta$, via the rule

$$
\begin{aligned}
\mathcal{T}_{\xi \eta} & =\delta_{\xi \eta} T_{\eta^{*} \eta^{*}}, \\
\mathcal{S}_{\xi \eta} & =\sum_{p \in \eta} S_{\xi^{*} p} .
\end{aligned}
$$

These matrices are well defined, i.e., independent of the choice of the representatives $\xi^{*} \in \xi$ (since $S^{2}$ commutes with both $T$ and $S$ ), and they determine a representation of $\mathrm{SL}_{2}(\mathbb{Z})$ which is trivial on the center: this is the reduced representation $\rho$. Note that all matrix elements of $\mathcal{S}$ are real numbers.

Some important properties of the modular representation $\varrho$ carry over to the reduced representation $\rho$ (e.g., the diagonality of the Dehn-twist $T$ ), while others (like the symmetry and unitarity of $S$ ) do not. The representation $\rho$ is equivalent to the largest subrepresentation of $\varrho$ trivial on $\left(\begin{array}{cc}-1 & 0 \\ 0 & -1\end{array}\right)$.

We note that, while the reduction process results clearly in loss of information, this loss is not as dramatic as one might expect: for example, it is possible to reconstruct from the knowledge of $\rho$ the charge conjugation and the real part of $S$, as well as the full matrix $T$.

\section{References}

[1] T.M. Apostol, Modular functions and Dirichlet series in number theory, 2nd edn, Springer, New York, 1990.

[2] A.O.L Atkin and H.P.F. Swinnerton-Dyer, Modular forms on noncongruence subgroups, Proc. Symp. Pure Math., 19, American Mathematical Society, Providence R.I., 1971, 1-26.

[3] P. Bántay, The kernel of the modular group representation and the Galois action in RCFT, Comm. Math. Phys. 233 (2003), 423.

[4] P. Bántay and T. Gannon, Conformal characters and the modular representation, JHEP 0602 (2006), 005.

[5] C.J. Cummins, Congruence subgroups of groups commensurable with PSL(2,Z) of genus 0 and 1, Experimt. Math. 13 (2004), 361-382.

[6] P. Di Francesco, P. Mathieu and D. Sénéchal, Conformal field theory, Springer, New York, 1997. 
[7] W. Eholzer and N.-P. Skoruppa, Modular invariance and uniqueness of conformal characters, Comm. Math. Phys. 174 (1995), 117-136.

[8] E. Hille, Lectures on ordinary differential equations, Addison-Wesley, Reading, Mass, 1969.

[9] J. Hurrelbrink, On presentations of $\mathrm{SL}_{n}\left(\mathbb{Z}_{s}\right)$, Comm. Algebra 11 (1983), 937-947.

[10] M.I. Knopp, Modular functions in analytic number theory, Markham, Chicago, 1970.

[11] M. Knopp and G. Mason, Vector-valued modular forms and Poincaré series, Illinois J. Math. 48 (2004), 1345-1366.

[12] S. Lang, Elliptic functions, 2nd edn, Springer, New York, 1987.

[13] S.P. Norton, More on moonshine, In: 'Computational group theory', ed. M.D. Atkinson, Academic Press, London, 1984, 185-193.

[14] S.P. Norton, Generalized moonshine, The Arcata Conference on Representations of Finite Groups, American Mathematical Society, Providence R.I., 1987, 208-209.

[15] G. Shimura, Introduction to the arithmetic theory of automorphic functions, Princeton University Press, Princeton, 1971.

[16] I. Tuba and H. Wenzl, Representations of the braid group $B_{3}$ and of $\mathrm{SL}(2, \mathbb{Z})$, Pacific J. Math. 197 (2001), 491-510.

[17] Y. Zhu, Modular invariance of characters of vertex operator algebras, J. Amer. Math. Soc. 9 (1996), 237-302.

Institute For TheOReticAl Physics

EÖTVÖS LORÁND UNIVERSITY

BUDAPEST

HUNGARY

E-mail address: bantay@general.elte.hu

Department of Mathematical Sciences

University of Alberta

EDMONTON

CANADA

E-mail address: tgannon@math.ualberta.ca

Received May 24, 2007; Accepted December 18, 2007 